\title{
Helping to address the over treatment of prostate cancer: tools to differentiate lethal and non-lethal phenotype in prostate cancer
}

\author{
Asian Journal of Andrology (2012) 14, 347-348; doi:10.1038/aja.2012.34; published online 23 April 2012
}

2 011 was a noteworthy year for those interested in the care of patients with prostate cancer. First, the US Preventive Services Task Force (USPSTF) released an update on prostate cancer screening, and recommended against the use of the prostate-specific antigen (PSA) test for all men, regardless of family history or race. While this surprised many in the field, the conclusion that there is more harm than benefit is inescapable on a population level-the focus of the USPSTF. Most men with newly diagnosed prostate cancer have favorable risk disease and are at an age when treatment is unlikely to extend life - a conclusion that is supported by randomized trials. Second, the National Institutes of Health convened a 'State of the Science' conference on active surveillance for prostate cancer, and concluded that the rates of overtreatment of prostate cancer are high and surveillance is greatly underutilized. Both the USPSTF and the National Institutes of Health conference were prompted in large part by the recognition that widespread PSA testing leads to prostate biopsies that often uncover indolent disease. In the majority of cases, treatment follows diagnosis, regardless of age or health status, leading to no improvement in length of life and a decline in quality of life. But in addition to the important issue of overdiagnosis and overtreatment, there is also evidence for undertreatment of aggressive prostate cancer. Both overtreatment and undertreatment are in part, the result of an inability to accurately characterize a heterogeneous disease with a generally long natural history. Current risk stratification paradigms underestimate and overestimate the potential risk that a newly diagnosed prostate cancer poses for an individual patient because of limitations in predicting the remaining years of life of the host, and the biological behavior of the cancer. In this issue of AJA, basic and clinical scientists discuss current and future approaches to improve the health outcomes of men who are diagnosed with prostate cancer.

As stated, the goal of this special issue is to determine if there are tools that can provide additional discrimination to the currently utilized clinical tools to identify those with the forms of prostate cancer most appropriate for a program such as active surveillance and those that will perhaps benefit for more aggressive therapeutic approaches. Two of the contributions take advantage of existing clinical data to determine if it is possible to utilize them in unique ways in order to provide such additive information. The manuscript of Nguyen and Kattan describes the utilization of clinical data to build prediction models that can then be evaluated to determine their current clinical capabilities. The manuscript by Dr Carter describes the utility of the kinetic patterns of PSA, as well as PSA isoforms and where they should be used in the current clinical paradigm of prostate cancer. Professor Kakehi in his article discusses the potential role(s) of active surveillance in the disease course of men with prostate cancer with particular focus on experiences in Japan.

In the manuscript by Dr Wilson, Dr Giovannucci and Dr Mucci, the authors examine lifestyle and dietary factor that may play a role in the prevention of more lethal prostate cancers. Of the tools that have been identified to provide discrimination of lethal prostate cancer, none have more support for their utilization than nuclear morphometry. In the article by Dr Veltri, Dr Christudass and Dr Isharwal, the authors describe the state of nuclear morphometric analyses in identifying aggressive prostate cancers, as well as complementary tools of analyzing nuclear proteins as molecular surrogates of the structural changes.

A majority of this special issue focuses on novel molecular approaches for the discrimination of lethal prostate cancer. The ability to decipher these types of molecular tools has been significantly advanced by the work of groups like those of Dr Jun Luo. In the manuscript by Dunn et al., they describe an analysis of approaches that are available to examine formal-fixed paraffin-embedded tissues and query them in molecular profiling studies. These approaches are providing for the ability to analyze samples with limited volume, such as prostatic biopsies, to provide for such signatures. The finding of the ETS gene rearrangements in prostate cancer was a fundamental discovery that has changed how we look at the disease. In the manuscript by Dr Rubin, he discusses the various types of both the ETS and nonETS gene rearrangements and their potential clinical significance as tools to provide a classification tool for prostate cancer. The cancer/ testis antigens (CTAs) are a family of proteins that, in the adult male, are normally limited in expression to the testes, but whose expression appears to be turned on in a number of cancer types. Dr Shiraishi, Dr Getzenberg and Dr Kulkarni have summarized the current understanding of the ability of CTAs to identify more aggressive and therefore lethal prostate cancer. Some of the CTA family members appear to be more highly expressed in lethal, metastatic disease whereas others appear to be turned off in the same men. Dr Terada and colleagues describe an extracellular matrix protein, Cyr61, that at the tissue level has been shown to be downregulated in more aggressive prostate cancer. They demonstrate an approach, utilizing commercially available antibodies, which can detect Cyr61 in the blood with potential to serve as a non-invasive tool for disease classification. In the manuscript by Dr Marcos and Dr Eeles, the authors describe our current 
understanding of two genes typically associated with breast cancer, $B R C A 1$ and $B R C A 2$. Their work has shown that germline mutations, particularly in the $B R C A 2$ gene may be a predictive factor for more aggressive prostate cancer. Finally, the contribution from Dr Isaacs, outlines our current understanding of the role that a genetic predisposition may play in the development of lethal forms of prostate cancer.

While this special issue describes a number of potential approaches that may provide tools that can add to our currently used clinical information, it seems that combinations of approaches and molecular profiles/markers will be necessary to truly provide predictive information. Disease and patient heterogeneity along with the multifocality with which the disease typically presents make this task seem daunting, but we are optimistic that such molecular discriminators may be utilized in the clinic in the not too distant future. We hope that this special issue sheds some light on this complex yet critically important clinical question.

Professor Robert H Getzenberg ${ }^{1}$ and Professor $\mathrm{H}$ Ballentine Carter ${ }^{2}$ ${ }^{1}$ Department of Urology, The Brady Urological Institute, Johns Hopkins Hospital,Baltimore, MD 21287, USA (rgetzen1@jhmi.edu) and ${ }^{2}$ Department of Urology, The Johns Hopkins University School of Medicine and The James Buchanan Brady Urological Institute, The Johns Hopkins Hospital, Baltimore, MD 21287, USA (carter.ballentine3@ gmail.com) 